\title{
Patterns of high-risk behaviors associated with HIV among male prisoners: A latent class analysis
}

\author{
Jalal Ale-Ebrahim ${ }^{1}$, Leila Janani ${ }^{2}$, Seyed Ahmad SeyedAlinaghi ${ }^{3}$, Behnam Farhoudi $^{4}$, Abbas Abbasi-Ghahramanloo ${ }^{5}$, \\ Mansoor Sajadipour ${ }^{6}$, Seyed Abbas Motevalian*7 (1)
}

Received: 14 Sep 2019

Published: 29 Aug 2020

\section{Abstract}

Background: Acquired Immunodeficiency Syndrome (AIDS) is one of the most important health challenges worldwide. Moreover, the prevalence of the Human Immunodeficiency Virus (HIV) infection differs among Iranian prisoners and the general population. The present study aimed to identify the patterns of HIV-related high-risk behaviors in male prisoners.

Methods: In this cross-sectional study, 2832 inmates were examined for HIV-related high-risk behaviors. The required data were collected using a questionnaire on high-risk behaviors, including a history of heterosexual and homosexual intercourse, and a history of drug use disorders. A Latent Class Analysis (LCA) was performed to analyze the obtained data in WinLTA software.

Results: Five latent classes were defined for the male prisoners, as follows: low-risk (20\%), moderate-risk (23\%), Injection Drug Use (IDU) (8\%), heterosexual intercourse/methamphetamine use (38\%), and high-risk (11\%). The LCA results revealed that high-risk sexual behaviors, IDU, and sharing injection equipment in prisons significantly influence the classification. The obtained data suggested that a history of imprisonment was not frequent in class $2(\mathrm{OR}=1.0033, \% 95 \mathrm{CI}: 0.9936-1.01)$ and class 4 members $(\mathrm{OR}=1.0053$, \%95CI:0.9929-1.0179). However, it was more prevalent in class 3 (OR=1.0164, \%95CI:1.0068-1.0262), and 5 $(\mathrm{OR}=1.0211, \% 95 \mathrm{CI}: 1.0129-1.0293)$. Heterosexual contact had the highest prevalence $(75 \%)$ in this regard. The results showed that the lowest prevalence of high-risk behaviors was associated with morphine use $(0.3 \%)$. Among illicit drugs, methamphetamine was the most prevalent drug $(42 \%)$ in the studied subjects.

Conclusion: The present study indicated a high prevalence of high-risk sexual behaviors and methamphetamine use in the investigated participants. We observed the co-occurrence of HIV-related high-risk behaviors in male prisoners. Thus, providing safe sex education for prisoners is highly recommended. It is also necessary to pursue care programs about IDU as a key risk factor for HIV transmission in prisons.

Keywords: Latent class analysis, Male prisoners, Male non-prisoner, Human Immunodeficiency Virus (HIV)

Conflicts of Interest: None declared

Funding: None

\section{*This work has been published underCC BY-NC-SA 1.0 license.}

Copyright $₫$ Iran University of Medical Sciences

Cite this article as: Ale-Ebrahim J, Janani L, SeyedAlinaghi SA, Farhoudi B, Abbasi-Ghahramanloo A, Sajadipour M, Motevalian SA. Patterns of high-risk behaviors associated with HIV among male prisoners: A latent class analysis.Med J Islam Repub Iran. 2020 (29 Aug);34:109. https://doi.org/10.47176/mjiri.34.109

\section{Introduction}

Prisoners are at risk of Human Immunodeficiency Virus (HIV) infection in Iran (1). Prisoners are at greater risk of

Corresponding author:DrSeyed Abbas Motevalian, motevalian.a@iums.ac.ir

1. Department of Epidemiology, School of Health, Iran University of Medical Sciences, Tehran, Iran

2. Department of Biostatistics, School of Public Health, Iran University of Medical Sciences, Tehran, Iran

3. Iranian Research Center for HIV/AIDS, Iranian Institute for Reduction of High-Risk Behaviors, Tehran University of Medical Sciences, Tehran, Iran

4. Social Determinants of Health Research Center, Amir-Almomenin Hospital, Tehran Medical Sciences Branch, Islamic Azad University, Tehran, Iran

5. Department of Public Health, School of Health, Ardabil University of Medical Sciences, Ardabil, Iran

6. Tehran University of Medical Sciences, Tehran, Iran

7. Research Center for Addiction and High-Risk Behaviors (ReCARB), Psychosocial Health Research Institute, Iran University of Medical Sciences, Tehran, Iran developing HIV, compared to other groups, globally. A combination of HIV, Drug Use Disorders (DUDs), and

$\uparrow$ What is "already known" in this topic:

In our study, the prevalence of substance abuse and some highrisk sexual behaviors were studied separately.

\section{$\rightarrow$ What this article adds:}

This study showed the co-occurrence of high-risk behaviors associated with HIV in prisoners. 
imprisonment threatens the health of communities (2).

HIV prevalence among prisoners is higher than the general population (3). In Iran, HIV prevalence among prisoners is 8 times higher than the general population (4). Its prevalence is approximately $1 \%$ in Iranian prisons (5). The prevalence of such infections could be related to various risk factors, such as a history of imprisonment, DUDs, drug injection, tattooing, having unprotected sex, using shared injection equipment, and deficient risk perception (6-8). Prisoners living with HIV could spread the infection in the general population (9).

Tehran, the capital city of Iran, with a population of 12800000 individuals (according to the last official census in 2016) is among the most populous cities in Iran. This population is prone to various difficulties if the necessary socioeconomic and cultural conditions are disregarded. Changes in the patterns of high-risk behaviors associated with HIV in the general population, as well as the risk of imprisonment, are among these difficulties.

Latent Class Analysis (LCA) is beneficial for classifying individuals. In this method, similar individuals are categorized within each class. This analysis defines different latent classes based on special indicators. LCA has been used in various studies on DUDs and sexual behaviors (10-12). The present study aimed to evaluate HIVrelated risky behaviors in male prisoners using an LCA. We also determined the effect of a history of imprisonment in different classes.

\section{Methods}

This cross-sectional study conducted including 2832 male inmates. The investigated prisoners' data were obtained from the database of Acquired Immunodeficiency Syndrome (AIDS) Research Center of Tehran University of Medical Sciences (TUMS). Moreover, the required data were collected from November 2013 to June 2014. The study participants were selected from a previous study on the prevalence of HIV infection among male prisoners in
Tehran (13). In total, 2840 cases were selected from the 6900 prisoners using Active Case Finding (ACF) technique. After clearing the data, 2832 of 2840 cases (18- to 65-year-olds) entered in this study.

The study was conducted using a questionnaire designed based on a form commonly used in Voluntary Counseling and Testing (VCT) centers in Iran. The necessary data were collected by a questionnaire containing information on high-risk behaviors, including heterosexual and homosexual intercourse, and the history of DUDs. Ten dichotomous variables were considered to assess risktaking behaviors related to HIV. The variables included the following: heterosexual and homosexual intercourse, a history of drug injection, as well as cannabis, opium, crack, methamphetamine, heroin, cocaine, and morphine use.

This study was approved by the ethics committee of Iran University of Medical Sciences (approval code: 2979, ethics code: IR.IUMS.REC.1397.707).All of the study participants' data were obtained anonymously to observe their confidentiality.

SPSS, Stata, and WinLTA software were implemented to achieve the pattern of high-risk behaviors. Latent class analysis is a form of modeling latent variables that defines the observed characteristics in heterogeneous populations into more homogeneous subtypes (14). The percentages of risky behaviors are presented in Table 1 . Furthermore, the $\mathrm{G}^{2}$, Akaike Information Criterion (AIC), and Bayesian Information Criterion (BIC) statistics were calculated to compare classes and to select the best model (Table 2).

\section{Results}

The mean \pm SD age of the study participants was $30.68 \pm 7.65$ years, with an age range of 18 to 65 years. Table 1 presents the distribution of characteristics associated with high-risk behaviors among the explored inmates.

To choose the best model, an LCA was conducted from class one to seven (Table 2). Among different models,

Table 1. Thefrequencyof prisoners with positive responses to the questions about risk-taking behaviors

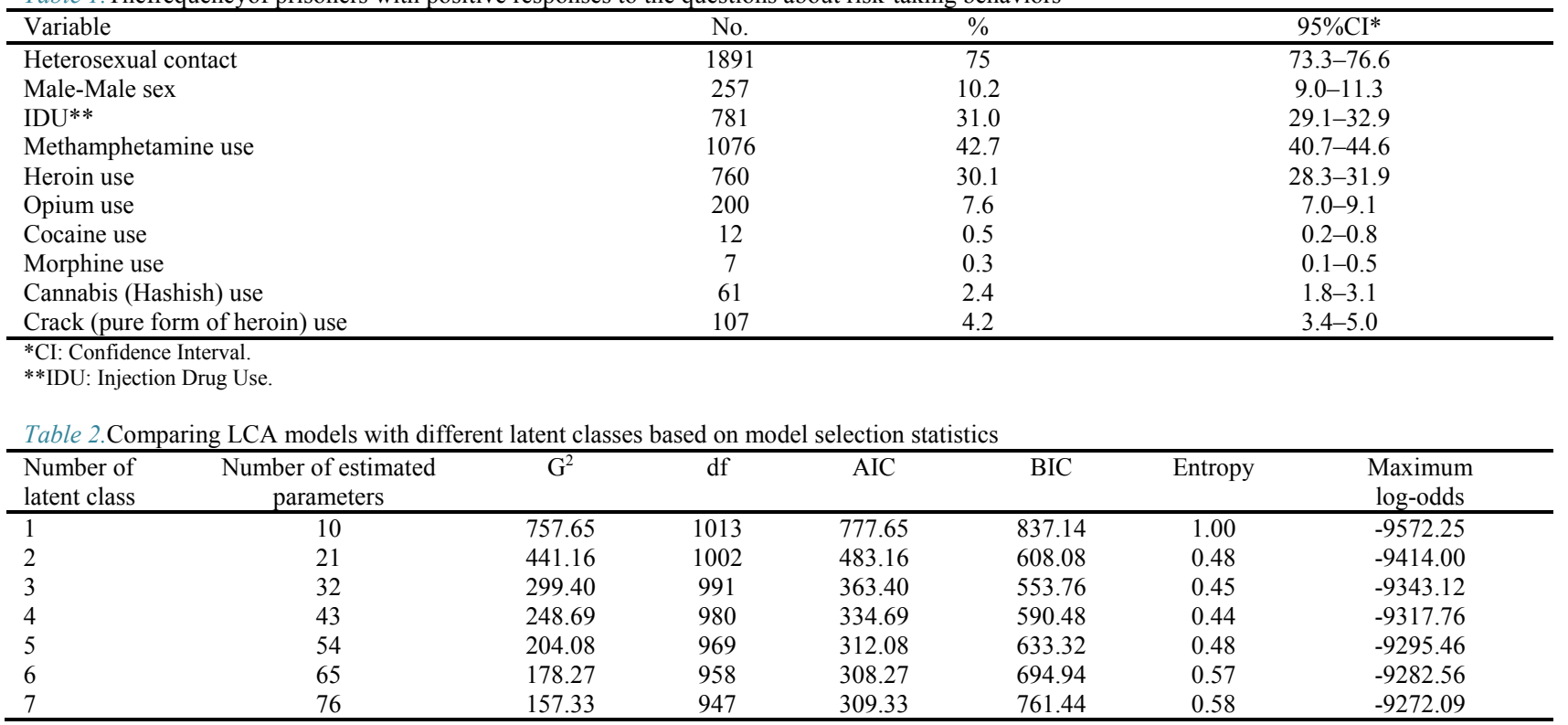




\begin{tabular}{|c|c|c|c|c|c|}
\hline & \multicolumn{5}{|c|}{ Latent class } \\
\hline & Low-risk & Moderate-risk & IDU & $\begin{array}{c}\text { Heterosexual/ } \\
\text { methamphetamine }\end{array}$ & High-risk \\
\hline $\begin{array}{l}\text { Latent class prevalence } \\
\text { Item-response probabilities }\end{array}$ & 0.2046 & 0.2282 & 0.0806 & 0.3815 & 0.1051 \\
\hline Heterosexual contact & 0.4957 & 0.6838 & 0.7200 & 0.8926 & 0.8440 \\
\hline Male-Male sex & 0.0264 & 0.0029 & 0.1838 & 0.1192 & 0.3026 \\
\hline IDU & 0.1618 & 0.4454 & 0.9518 & 0.0457 & 0.6034 \\
\hline Methamphetamine use & 0.1377 & 0.4461 & 0.0222 & 0.5334 & 0.6671 \\
\hline Heroin use & 0.0266 & 0.6399 & 0.3359 & 0.0889 & 0.7499 \\
\hline Opium use & 0.2374 & 0.0070 & 0.0003 & 0.0391 & 0.1600 \\
\hline Cocaine use & 0.0039 & 0.0001 & 0.0000 & 0.0000 & 0.0326 \\
\hline Morphine use & 0.0079 & 0.0000 & 0.0039 & 0.0014 & 0.0000 \\
\hline Cannabis (Hashish) use & 0.0005 & 0.0000 & 0.0000 & 0.0503 & 0.0314 \\
\hline Crack(pure form of heroin) use & 0.0065 & 0.0005 & 0.1560 & 0.0138 & 0.2094 \\
\hline Covariates & Reference & OR $(95 \% \mathrm{CI})$ & OR $(95 \% \mathrm{CI})$ & OR $(95 \% \mathrm{CI})$ & OR $(95 \% \mathrm{CI})$ \\
\hline Age, $y(p \leq 0.001)$ & Reference & $\begin{array}{c}0.3280 \\
(0.1404-0.7663)\end{array}$ & $\begin{array}{c}0.2975 \\
(0.1300-0.6805)\end{array}$ & $\begin{array}{c}0.0319 \\
(0.0140-0.0725)\end{array}$ & $\begin{array}{c}0.6464 \\
(0.2125-0.9660)\end{array}$ \\
\hline Prison history $(\mathrm{p} \leq 0.001)$ & Reference & $\begin{array}{c}1.0033 \\
(0.9936-1.0132)\end{array}$ & $\begin{array}{c}1.0164 \\
(1.0068-1.0262)\end{array}$ & $\begin{array}{c}1.0053 \\
(0.9929-1.0179)\end{array}$ & $\begin{array}{c}1.0211 \\
(1.0129-1.0293)\end{array}$ \\
\hline
\end{tabular}

Note: The probability of a "No" response can be calculated by subtracting the item-response probabilities demonstrated above.

*Item-response probabilities of $>0.5$ are in bold to facilitate interpretation.

based on the $\mathrm{G}^{2}$, BIC, and AIC values, as well as the interpretability of the model, the authors should select the best one. Eventually, a 5-class model was developed as the final model. The results of the analysis of the 5-class model are presented in Table 3. The first section of this table describes the prevalence of each class. In the second part, the probability of engaging in each risk-related behavior per class was specified. These probabilities were the basis for the interpretation and labeling of classes.

The latent classes were named as follows: low risk $(20 \%)$, moderate risk $(23 \%)$, IDU ( $8 \%)$, heterosexual intercourse/methamphetamine use (38\%), and high risk (11\%). The smallest class belonged to class 3 (IDU) with a prevalence rate of $8 \%$ and an IDU history of approximately $100 \%$. The largest category was class 4 , with a prevalence rate of $38 \%$. In this class, individuals were more likely to have heterosexual intercourse under the methamphetamine use effect.

The Odds Ratio (OR) indicated the odds of membership per class, compared to the low-risk (first-class) group. As per Table 3, for every year increase in age, the odds of membership in each class decreased, compared to the first class. Additionally, a history of imprisonment on the odds of membership in classes 2 and 4 was ineffective; however, it increased the odds of membership in classes 3 (IDU) and 5 (high-risk).

\section{Discussion}

In the current study, using LCA, the patterns of highrisk behaviors associated with HIV based on observed variables were identified. Among all assessed high-risk behaviors in this study, heterosexual intercourse had the highest prevalence rate $(75 \%)$.

The high prevalence of heterosexual behavior in prisoners compared to the general population has also been reported in previous studies (15-17). The lowest prevalence rate in the present study belonged to morphine use $(0.3 \%)$.
Among the drugs, methamphetamine use was the most frequent substance with $42 \%$; this finding is compatible with that of a study conducted in another country (18). Another study in Iran reported a higher prevalence rate of other substances (19) in this population. In this study, in class 1 (low-risk) with a prevalence of $20 \%$, all HIVassociated risk factors had a very low probability. In class 2 (moderate risk) with a prevalence of $23 \%$, having heterosexual intercourse and heroin use had the highest probability.

In class 2 , although using opium, crack, cannabis, morphine, and cocaine were uncommon, but these substances were used through injection. Class 3 (IDU) data with a prevalence of $8 \%$ were compatible with those of another study (20). Class 4 had a prevalence rate of $38 \%$, with high odds of having heterosexual contact and methamphetamine use. While some studies highlighted the cooccurrence of methamphetamine use and high-risk sexual behaviors, both of which increase the probability of HIV infection $(21,22)$. Class 4 had a high probability of heterosexual and methamphetamine use. Homosexual contact had a $30 \%$ probability in class 5 that is the highest rate among all latent classes.

Our results indicated that having heterosexual intercourse affected the clustering of study participants significantly. In other words, this behavior was highly prevalent in all latent classes. People Living With HIV (PLWH) are usually sexually-active and high-risk sexual behaviors are frequently reported in them (23). As a result, sexuallyactive prisoners who are HIV positive could significantly impact the spread of this infection.

Due to the lack of similar studies on implementing of LCA in high-risk HIV-related behaviors, considering different indicators and the lack of comparability, the findings of some of these investigations are difficult to be understood.

In a study on the acceptability of HIV testing in those exposed to high levels of HIV infection by an LCA (24), a 
4-class model was proposed. Classes were as follows: low-risk $(\mathrm{n}=324,21.6 \%)$, concurrent multiple sexual partners $(\mathrm{n}=346 ; 23.0 \%)$, prisoners and substance users $(\mathrm{n}=293 ; 19.5 \%)$, and having unprotected sex/with multiple sexual partners $(n=538 ; 35.8 \%)$. In this study, concurrent multiple sex partners were the most common practice $(61 \%)$ in one class (class 2$)$. The class 3 of the study presented high odds of alcohol and substance use; $75 \%$ of them reported cocaine or crack use, $43 \%$ reported another drug use, and $34 \%$ were exposed to HIV infection. The class 4 in the above-mentioned study was the largest class with a probability of $35 \%$. Besides, the odds of having unprotected sex in this class were $79 \%$, and $30 \%$ of them had 2-5 sexual partners.

A study investigated the pattern of HIV-related factors in injection drug users in Kermanshah (25). Subsequently, a 3-class model was generated as the most appropriate model in which the high-risk class had a 33\% probability. In addition, in this class, a relatively high percentage $(54 \%)$ of individuals used shared injection equipment, and $63 \%$ reported having unprotected sex.

In our study, a history of being in prison increased the odds of membership in the IDU class $(\mathrm{OR}=1.0053$, 95\%CI: 1.0068-1.0262). This finding was consistent with that of another international research (11).

As per the previous studies, the risk of sex-related highrisk behaviors and substance use among prisoners was higher than that of the general population.

In our study, data on prisoners explored in 2014 were used to facilitate the research process, as it required a long time to gather information from prisoners. The pattern of high-risk behaviors in prisoners may have varied from 2014 to 2018; thus, it cannot be demonstrated in this study. The cross-sectional nature of the current study also prevented the researchers from providing a causal interpretation of the achieved data.

\section{Conclusion}

The obtained data suggested the co-occurrence of HIVrelated high-risk behaviors in he investigated male prisoners through a 5-class model. A sexual relationship with the opposite sex in male inmates plays an essential role in classifying individuals; therefore, providing more effective education on a safe-sex is required for inmates. Despite all the efforts, injection drug use significantly impacts the classification of inmates. Accordingly, it is necessary to have IDU care programs as a key risk factor for HIV transmission.

\section{Acknowledgment}

We would like to thank the Iranian Research Center for HIV/AIDS (IRCHA) affiliated to Tehran University of Medical Sciences (TUMS) and the prison staff, who assisted in data collection.

\section{Conflict of Interests}

The authors declare that they have no competing interests.

\section{References}

1. Sharifi H, Mirzazadeh A, Shokoohi M, Karamouzian M, Khajehkazemi R, Navadeh S, et al. Estimation of HIV incidence and its trend in three key populations in Iran. PloS One. 2018;13(11):e0207681

2. Akbari M, Akbari M, Naghibzadeh-Tahami A, Joulaei H, Nasiriyan M, Hesampour M, et al. Prevalence of HIV/AIDS among Iranian prisoners: a review article. Addict Health. 2016;8(3):195.

3.Jürgens R, Nowak M, Day M. HIV and incarceration: prisons and detention. J Int AIDS Soc. 2011;14(1):26.

4.S hahbazi M, Farnia M, Moradi G, Ebrahimi B. The trend of HIV/AIDS prevalence among IDU's in Iranian prisoners (13761386). Retrovirology. 2010;7(S1):P101.

5.Seyedalinaghi SA, Farhoudi B, Mohraz M, Firouzeh MM, Hosseini M, Kamali K. Prevalence of HIV in a Prison of Tehran by Active Case Finding. Iran J Public Health. 2017;46(3):431-2.

6. Ravlija J, Vasilj I, Marijanović I, Vasilj M. Risk behaviour of prison inmates in relation to HIV/STI. Psychiatr Danub. 2014;26(Suppl 2):395-401.

7. Kheirandish P, Seyedalinaghi S, Hosseini M, Jahani M, Shirzad H, Foroughi M, et al. Prevalence and correlates of HIV infection among male injection drug users in detention in Tehran, Iran. J Aquir Immune Defic. 2010;53(2):273-5.

8. Khajehkazemi R, Haghdoost A, Navadeh S, Setayesh H, Sajadi L, Osooli M, et al. Risk and vulnerability of key populations to HIV infection in Iran; knowledge, attitude and practises of female sex workers, prison inmates and people who inject drugs. Int J Sex Health. 2014;11(6):568-74

9. Epperson MW, Khan MR, El-Bassel N, Wu E, Gilbert L. A longitudinal study of incarceration and HIV risk among methadone maintained men and their primary female partners. AIDS Behav. 2011;15(2):347-55

10. McCarty-Caplan D, Jantz I, Swartz J. MSM and drug use: a latent class analysis of drug use and related sexual risk behaviors. AIDS Behav. 2014;18(7):1339-51.

11. Noor SW, Ross MW, Lai D, Risser JM. Use of latent class analysis approach to describe drug and sexual HIV risk patterns among injection drug users in Houston, Texas. AIDS Behav. 2014;18(3):276-83.

12. Tobin KE, Yang C, King K, Latkin CA, Curriero FC. Associations between drug and alcohol use patterns and sexual risk in a sample of African American men who have sex with men AIDS Behav. 2016;20(3):590-9.

13. Golsoorat F, Pahlaviani M. Prevalence and associated factors of HIV infection among male prisoners in tehran, Iran. Arch Iran Med. 2017;20(6):356.

14. Azar P, Wood E, Nguyen P, Luma M, Montaner J, Kerr T, et al. Drug use patterns associated with risk of non-adherence to antiretroviral therapy among HIV-positive illicit drug users in a Canadian setting: a longitudinal analysis. BMC Infect Dis. 2015;15(1):193.

15. Farhoudi B, SeyedAlinaghi S, Ataeinia B, Hosseini M, Jafari S, Alasvand R, et al. Comparison of HIV indicators after implementation of a new model for TB and HIV management in an Iranian prison. Infect Disord Drug Targets. 2019.

16. Ghalehkhani N, Farhoudi B, Gouya MM, Sharifi H, SeyedAlinaghi $\mathrm{S}$, Kamali K, et al. The HIV treatment cascade in people living with HIV in Iran in 2014: Mixed-method study to measure losses and reasons. Int J STD AIDS. 2019:0956462419867573.

17. Golrokhi R, Farhoudi B, Taj L, Pahlaviani FG, Mazaheri-Tehrani E, Cossarizza A, et al. HIV Prevalence and Correlations in Prisons in Different Regions of the World: A Review Article. Open AIDS J. 2018;12:81.

18. Goodman-Meza D, Shoptaw S, Weiss RE, Nakazono T, Harawa NT, Takada S, et al. Methamphetamine use drives decreases in viral suppression for people living with HIV released from a large municipal jail: Results of the LINK LA clinical trial. Drug Alc dep. 2019;202:178-84

19. Khalooei A, Mashayekhi-Dowlatabad M, Rajabalipour MR, Iranpour A. Pattern of substance use and related factors in male prisoners. Addict Health. 2016;8(4):227.

20. Moradi G, Darvishi S, Asaadi L, Zavareh FA, Gouya MM, Tashakorian M, et al. Patterns of Drug Use and Related Factors Among Prisoners in Iran: Results from the National Survey in 2015. J Prim Prev. 2020:1-10. 
21. Hittner JB. Meta-analysis of the association between methamphetamine use and high-risk sexual behavior among heterosexuals. Psychol Behav Addict .2016;30(2):147.

22. Liu D, Jiang Z, Xiu C, Li Z, Song Q, Wang Z. Sexually transmitted infection prevalence and related risk factors among heterosexual male methamphetamine users in China. Int $\mathrm{J}$ STD AIDS. 2017;28(12):1208-14

23. Du P, Crook T, Whitener C, Albright P, Greenawalt D, Zurlo J. HIV transmission risk behaviors among people living with HIV/AIDS: The need to integrate HIV prevention interventions and public health strategies into HIV care. J Public Health Manag Pract. 2015;21(2):E1.

24. Nunn A, Brinkley-Rubinstein L, Rose J, Mayer K, Stopka T, Towey $\mathrm{C}$, et al. Latent class analysis of acceptability and willingness to pay for self-HIV testing in a United States urban neighbourhood with high rates of HIV infection. J Int Soc. 2017;20(1):21290.

25. Sharifi H, Mirzazadeh A, Noroozi A, Marshall BD, Farhoudian A, Higgs $\mathrm{P}$, et al. Patterns of HIV risks and related factors among people who inject drugs in Kermanshah, Iran: a latent class analysis. J Psyco act. 2017;49(1):69-73. 